ApJ AcCepted 6 February 2018

Preprint typeset using $\mathrm{LAT}_{\mathrm{E}} \mathrm{X}$ style emulateapj v. 01/23/15

\title{
H I KINEMATICS ALONG THE MINOR AXIS OF M82
}

\author{
Paul Martini ${ }^{1,2}$, Adam K. Leroy ${ }^{1}$, Jeffrey G. Mangum ${ }^{3}$, Alberto Bolatto ${ }^{4}$, Katie M. Keating ${ }^{5}$, Karin \\ SANDSTROM $^{6}$, FABian WALTER ${ }^{7}$ \\ ApJ Accepted 6 February 2018
}

\begin{abstract}
M82 is one of the best studied starburst galaxies in the local universe, and is consequently a benchmark for studying star formation feedback at both low and high redshift. We present new VLA H I observations that reveal the cold gas kinematics along the minor axis in unprecedented detail. This includes the detection of $\mathrm{H} \mathrm{I}$ up to $10 \mathrm{kpc}$ along the minor axis toward the South and beyond $5 \mathrm{kpc}$ to the North. A surprising aspect of these observations is that the line-of-sight H I velocity decreases substantially from about $120 \mathrm{~km} \mathrm{~s}^{-1}$ to $50 \mathrm{~km} \mathrm{~s}^{-1}$ from 1.5 to $10 \mathrm{kpc}$ off the midplane. The velocity profile is not consistent with the $\mathrm{H}$ I gas cooling from the hot wind. We demonstrate that the velocity decrease is substantially greater than the deceleration expected from gravitational forces alone. If the $\mathrm{H}$ I consists of a continuous population of cold clouds, some additional drag force must be present, and the magnitude of the drag force places a joint constraint on the ratio of the ambient medium to the typical cloud size and density. We also show that the H I kinematics are inconsistent with a simple conical outflow centered on the nucleus, but instead require the more widespread launch of the $\mathrm{H} I$ over the $\sim 1 \mathrm{kpc}$ extent of the starburst region. Regardless of the launch mechanism for the H I gas, the observed velocity decrease along the minor axis is sufficiently great that the H I may not escape the halo of M82. We estimate the $\mathrm{H}$ I outflow rate is much less than $1 \mathrm{M}_{\odot} \mathrm{yr}^{-1}$ at $10 \mathrm{kpc}$ off the midplane.
\end{abstract}

Keywords: galaxies: individual(M82 - galaxies: ISM - galaxies: starburst - intergalactic medium ISM: jets and outflows - ISM: molecules

\section{INTRODUCTION}

Galaxies with substantial star formation rates often have large-scale winds that represent material flowing out of the galaxy along the minor axis (e.g. Heckman et al. 1990; Veilleux et al. 2005). These winds are most likely driven by young, massive stars, which have substantial stellar winds and explode as core-collapse supernovae. The material in these winds can entrain all phases of the interstellar medium, and is expected to be preferentially metal-enriched by the nucleosynthetic products in supernova ejecta. If the material in these winds escapes the galaxy, the winds can enrich the circumgalactic and intergalactic medium (e.g. Borthakur et al. 2013; Werk et al. 2016) and regulate the growth and metal-enrichment histories of galactic disks (e.g. Oppenheimer \& Davé 2008; Peeples \& Shankar 2011). Observational evidence of the removal of metal-encirched material includes the metallicity of the intergalactic medium, the mass-metallicity relationship, and the sizes and luminosities of galactic disks.

\footnotetext{
${ }^{1}$ Department of Astronomy, The Ohio State University, Columbus, OH 43210, USA, martini.10@osu.edu, leroy.42@osu.edu

2 Center for Cosmology and Astroparticle Physics, The Ohio State University, Columbus, OH 43210, USA

${ }^{3}$ National Radio Astronomy Observatory, 520 Edgemont Road, Charlottesville, VA 22903, USA

${ }^{4}$ Department of Astronomy, University of Maryland, College Park, MD 20742, USA

${ }^{5}$ Rincon Research Corporation, 101 North Wilmot Road, Suite 101, Tucson, AZ 85711, USA

${ }^{6}$ Department of Physics, University of California, San Diego, CA 92093, USA

7 Max-Planck-Institute für Astronomie, Königstuhl 17, D-69117 Heidelberg, Germany
}

M82 is arguably one of the best-studied galaxies with a starburst-driven wind (Lvnds \& Sandage 1963). Its two key properties are its proximity at only $D=3.63$ Mpc (Freedman et al. 1994; Gerke et al. 2011) in the M81 group and that the galaxy is observed nearly edge on, which makes it particularly well-suited to observe the outflow along the minor axis. Studies of this galaxy across the electromagnetic spectrum have detected multiple phases of the wind moving at multiple velocities, ranging from plasma temperatures of $30-80$ million Kelvin at X-ray energies (Schaaf et al. 1989; Strickland \& Heckman 2009) to warm, ionized gas traced by visible-wavelength features such as $\mathrm{H} \alpha$ (McKeith et al. 1995; Westmoquette et al. 2009) to atomic H I and molecular gas at temperatures of $100 \mathrm{~K}$ or less (Seaquist \& Clark 2001; Walter et al. 2002; Salak et al. 2013; Lerov et al. 2015).

Observations of visible emission lines along the minor axis showed line splitting in position velocity diagrams (Axon \& Taylor 1978; Amirkhanyan et al. 1982), and subsequent Fabry-Perot data exhibited good evidence for a large-scale, biconical outflow (Bland \& Tully 1988). The region with double-peaked emission lines begins about $300 \mathrm{pc}$ from the midplane and has been detected to about $1 \mathrm{kpc}$ (Heckman et al. 1990). McKeith et al. (1995) derived an inclination of $80^{\circ}$ for the galactic disk, and a cone opening angle of $30^{\circ}$ (semi-angle $15^{\circ}$ ) at $>1$ kpc. Their model starts as a cylindrical flow and transitions to a bicone at about $300 \mathrm{pc}$, and then radially accelerates to a terminal velocity of $600 \mathrm{~km} \mathrm{~s}^{-1}$ on the surface of the bicone. The material inside the bicone is the much hotter fluid observed at X-ray energies and predicted to have a terminal velocity several times greater than the $\sim 500 \mathrm{~km} \mathrm{~s}^{-1}$ escape velocity Strickland \& Heckman 
2009)

The line of sight velocities of $120-140 \mathrm{~km} \mathrm{~s}^{-1}$ relative to systemic for the cold atomic and molecular gas traced by $\mathrm{H}$ I and $\mathrm{CO}$ are substantially slower than the emission traced at shorter wavelengths. The cold gas could be entrained in the hot gas, although simulations suggest that only a small fraction of the cold gas should survive a significant amount of acceleration (Scannapieco \& Brüggen 2015; Schneider \& Robertson 2017), except perhaps if the clouds are supported by magnetic fields (McCourt et al. 2015). Another possibility is that the winds are driven by radiation pressure through momentum transfer from starlight to dust grains embedded in the gas (Murray et al. 2005, 2010; Andrews \& Thompson 2011). Recent hydrodynamic simulations by Zhang et al. (2017) suggest that clouds accelerated by the radiation field last significantly longer than clouds entrained in the hot outflow. Both the atomic and molecular gas show double-peaked lines that suggest the hot, conical outflow is bounded by a sheath of cooler material (Walter et al. 2002). Lerov et al. (2015) conclude that the cold $\mathrm{H}$ I and CO help to confine the hot outflow. The best evidence for this connection begins approximately $1.5 \mathrm{kpc}$ from the disk. At these distances the $\mathrm{H} \mathrm{I}$ and $\mathrm{CO}$ exhibit double-peaked profiles indicative of a bicone, a velocity gradient consistent with the outflow, and spatial coincidence with features at other wavelengths (see Figure 16 in Leroy et al. 2015).

In this paper we present new, wide-field H I observations of M82 obtained with the Karl G. Jansky Very Large Array (VLA) combined with previous observations with the 100-m Robert C. Byrd Green Bank Telescope (GBT). These observations show the $\mathrm{H}$ I intensity and kinematics over approximately $20^{\prime} \times 20^{\prime}$ with a resolution of $24^{\prime \prime}$. Based on the distance $D=3.63 \mathrm{Mpc}, 1 \mathrm{kpc} \approx 1^{\prime}$. These observations therefore trace the $\mathrm{H}$ I outflow up to a projected distance of $10 \mathrm{kpc}$ along the minor axis. The next section describes our observations in further detail, and the following section presents a mass model for M82. We use this mass model in 44 to explore various explanations for the minor axis kinematics, and summarize our results in 95 . Except where otherwise noted, we adopt an inclination of $80^{\circ}$ for the disk of M82 (Lynds \& Sandage 1963; McKeith et al. 1993).

\section{OBSERVATIONS}

We observed M82 in the L band with the VLA in the B, C, and D configurations in 2015 and 2016, and combined these data with $\mathrm{L}$ band observations from the GBT obtained between 2003 and 2009. The VLA observations were centered on M82 at $\alpha=9: 55: 52.72, \delta=+69: 40: 45.7$ (J2000), and on-source integration times in the B, C, and $\mathrm{D}$ configurations were $\sim 378,294$, and 168 minutes, respectively. The GBT observations consist of 40 sessions that include the M81/M82 and NGC2403 galaxy groups. The GBT observations were used to construct an $8.7^{\circ} \times 21.3^{\circ}$ map centered at $\alpha=8: 40: 37.0, \delta=$ $+69: 17: 16$ (J2000) and have a total integration time of approximately 187 hours. This GBT map includes data previously described in Chynoweth et al. 2008, 2009, 2011).
We set the VLA correlator to observe the full, polarized L-band continuum with $1 \mathrm{MHz}$ channels across the full range $\sim 1-2 \mathrm{GHz}$. We also configured spectral windows to target the $\mathrm{H} \mathrm{I}$ and $\mathrm{OH}$ lines at higher spectral resolution. For the $\mathrm{H}$ I line, which is the focus of this paper, we used $20483.906 \mathrm{kHz}$ channels centered on the $\mathrm{H}$ I line at the velocity of M82. For the $\mathrm{OH}$ lines, we used a slightly coarser channel width of $7.812 \mathrm{kHz}$. We calibrated the amplitude, bandpass, and phase with measurements of $1331+305$ (3C 286; amplitude and bandpass; flux density $=15.0 \mathrm{Jy}$ at $1.4 \mathrm{GHz}$ ) and $\mathrm{J} 0921+6215$ (B-configuration phase; flux density $=1.186 \pm 0.003 \mathrm{Jy} /$ beam at $1.55 \mathrm{GHz}$ ), and J0841 + 7053 (C- and D-configuration phase; flux density $=3.340 \pm 0.018 \mathrm{Jy} /$ beam at $1.57 \mathrm{GHz}$ ), respectively, using standard techniques. This observation strategy mirrored that used successfully for THINGS, VLA ANGST, and LITTLE THINGS (Walter et al. 2008; Hunter et al. 2012; Ott et al. 2012). While M82 was extensively observed by the VLA in the 1980s (Yun et al. 1993, 1994), the current observations are more sensitive, have higher velocity resolution, and have much better dynamic range due to upgrades from the early 1990s to late 2000s that improved sensitivity and bandwidth. The later is particularly important, given M82's substantial continuum emission.

As the continuum emission from M82 is quite strong, we self-calibrated all measurements. The reduction in phase residual following self-calibration resulted in a factor of three to five improvement in peak signal-to-noise for all measurements. After self-calibration, we split out the spectral window containing $\mathrm{H} \mathrm{I}$, binning the data to have $8 \mathrm{~s}$ integrations. We identified line free channels from the integrated spectrum of the $u-v$ data. We then fit and subtracted a first order polynomial from each visibility measurement. We combined all of the continuum-subtracted visibility data for all four observations, in the process binning the data to a final channel width of $5 \mathrm{~km} \mathrm{~s}^{-1}$.

We imaged the data in stages to account for the substantial absorption in the center of M82, . First, we created a high resolution image with Briggs weighting parameter robust $=0$ and no $u-v$ taper. This image had a beam size of $\sim 5^{\prime \prime}$. The surface brightness sensitivity of this image was too poor to study the outflow in detail, but yielded a good initial model of the inner part of the galaxy. We cleaned in interactive mode until the maximum residual appeared comparable to the noise in the image. We then imaged the data again, beginning from the model output by the previous run, and this time used robust $=0.5$ weighting to emphasize surface brightness sensitivity slightly more. Again, we cleaned until the maximum residual was comparable to the noise. Then, starting from the model output by that imaging run, we imaged the data with an $18^{\prime \prime} u-v$ taper. This dramatically improved the surface brightness sensitivity of the data. Because this step began with the previous, higherresolution model, we achieved a substantial improvement in surface brightness sensitivity without significant, negative impact due to the strong absorption in the center. We cleaned this image until the maximum residual again resembled the noise of the image and smoothed the output image to have a round, $24^{\prime \prime}$ beam (FWHM). 

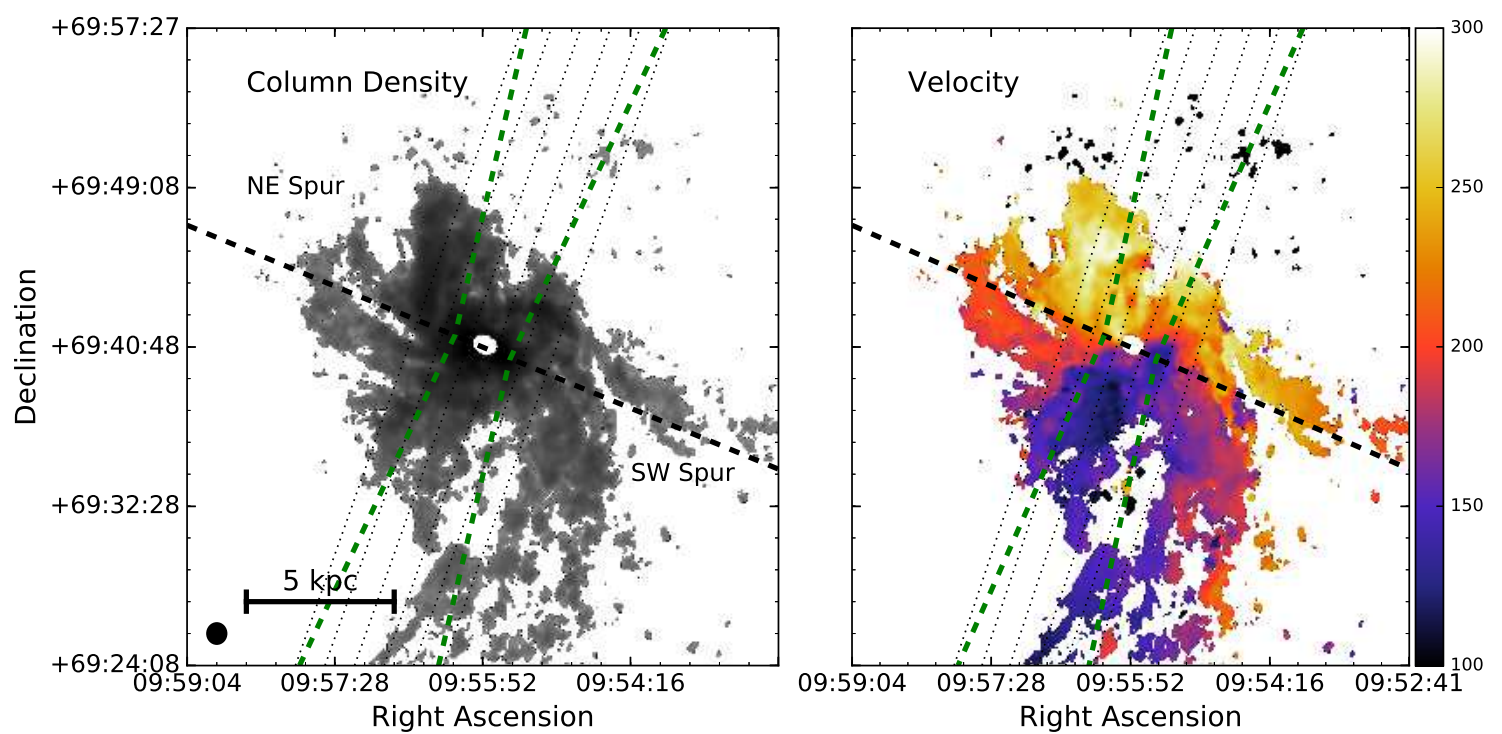

Figure 1. Log of the M82 H I column density (left) and velocity field (right). The velocity scale in $\mathrm{km} \mathrm{s}^{-1}$ is shown on the right colorbar. The major axis is oriented at $P A=67^{\circ}$ (black, dashed line). The outline of our biconical frustum model (green, dashed line) is oriented along the minor axis with an opening angle of $\theta=20^{\circ}$ (see $\S 4.2$ ). Also shown are the outlines of various 1 kpc wide slices along the minor axis used to compute position-velocity diagrams and a scale bar with a projected length of $5 \mathrm{kpc}$. The absence of $\mathrm{H} \mathrm{I}$ at the center position is because there is $\mathrm{H}$ I absorption of the bright continuum source.

\subsection{GBT Data Processing}

The M81/M82 and NGC 2403 area was observed by moving the telescope in declination and sampling every $3^{\prime}$, with an integration time of 1-3 seconds per sample (the integration time varied with the observation session). Strips of constant declination were spaced by $3^{\prime}$, and the telescope was moved in right ascension to form a basket weave pattern over the region. The GBT spectrometer was used with a bandwidth of either 12.5 or 50 $\mathrm{MHz}$, depending on the observation session. The combined bandwidth for the final map is $10.5 \mathrm{MHz}$, and has a velocity range from -890 to $1320 \mathrm{~km} \mathrm{~s}^{-1}$. The typical system temperature for each channel of the dualpolarization receiver was $\approx 20 \mathrm{~K}$.

The GBT data were reduced in the standard manner using the GBTIDL and AIPS data reduction packages. Spectra were smoothed to a channel spacing of $24.4 \mathrm{kHz}$, corresponding to a velocity resolution of $5.2 \mathrm{~km} \mathrm{~s}^{-1}$. A reference spectrum for each of the nine observation sessions was made from an observation of an emission-free region, usually from the edges of the maps. The reference spectrum was used to perform a (signal-reference)/reference calibration of each pixel. These calibrated spectra were scaled by the system temperature and corrected for atmospheric opacity and GBT efficiency. We adopted the GBT efficiency from equation one of Langston \& Turner (2007) for a zenith atmospheric opacity of $\tau_{0}=0.009$. The frequency range observed was relatively free of radio frequency interference (RFI) and less than $0.3 \%$ of all spectra were adversely affected. The spectra exhibiting RFI were identified by tabulating the root-mean-square (RMS) noise level in channels free of neutral hydrogen emission. Spectra that showed unusually high noise across many channels were flagged and removed. The observations were then gridded using the AIPS task SDIMG, which also averages polarizations. After amplitude calibration and gridding, a 1st-order polynomial was fit to line-free regions of the spectra and subtracted from the gridded spectra using the AIPS task IMLIN. The effective angular resolution, determined from maps of $3 \mathrm{C} 286$, is $9.15 \pm 0.05^{\prime}$. To convert to units of flux density, we observed the calibration source $3 \mathrm{C} 286$. The calibration from Kelvin to Janskys was derived by mapping $3 \mathrm{C} 286$ in the same way that the HI maps were produced, and the scale factor from $\mathrm{K} /$ Beam to Jy/Beam is $0.43 \pm 0.03$. Due to the patchwork nature of the observations, the RMS noise varies considerably across the datacube, ranging between $6-30$ $\mathrm{mJy} /$ beam. The average RMS noise in the final data cube is $20 \mathrm{mJy}$ per $24.4 \mathrm{kHz}$ channel.

\subsection{Construction of Combined Data Products}

The VLA-only cube still exhibits "bowling" and other large scale artifacts, in part due to the absence of short and zero spacing data. To deal with this, we combined the VLA cube with the GBT cube. First, we extracted a subcube centered on M82 from the larger M81 GBT survey. We then masked channels dominated by Galactic emission, carried out one additional round of first-order baseline fitting, applied the primary beam taper of the VLA data to the GBT data, and combined the VLA and GBT data with the CASA task feather. This combination removed or suppressed most of the large scale artifacts that were present in the VLA-only cube. After the combination, we corrected the cube for the primary beam response of the VLA. The final cube has a channel width of $5 \mathrm{~km} \mathrm{~s}^{-1}$, beam size of $24^{\prime \prime}$ (FWHM), and covers the primary beam of the VLA out to its half power point $\left(\approx 32^{\prime}\right)$. Before the primary beam correction, the cube had an rms noise of $\approx 0.4$ mJy beam $^{-1}$ at this resolution and channel width, equivalent to $\approx 4 \mathrm{~K}$ noise for $\approx 1050 \mathrm{~K} / \mathrm{Jy}$.

We converted the cube to have units of Kelvin, and also made a version of our $\mathrm{H}$ I cube with individual channels in units of column density. To do this we used the $5 \mathrm{~km} \mathrm{~s}^{-1}$ width of each channel and assumed optically 


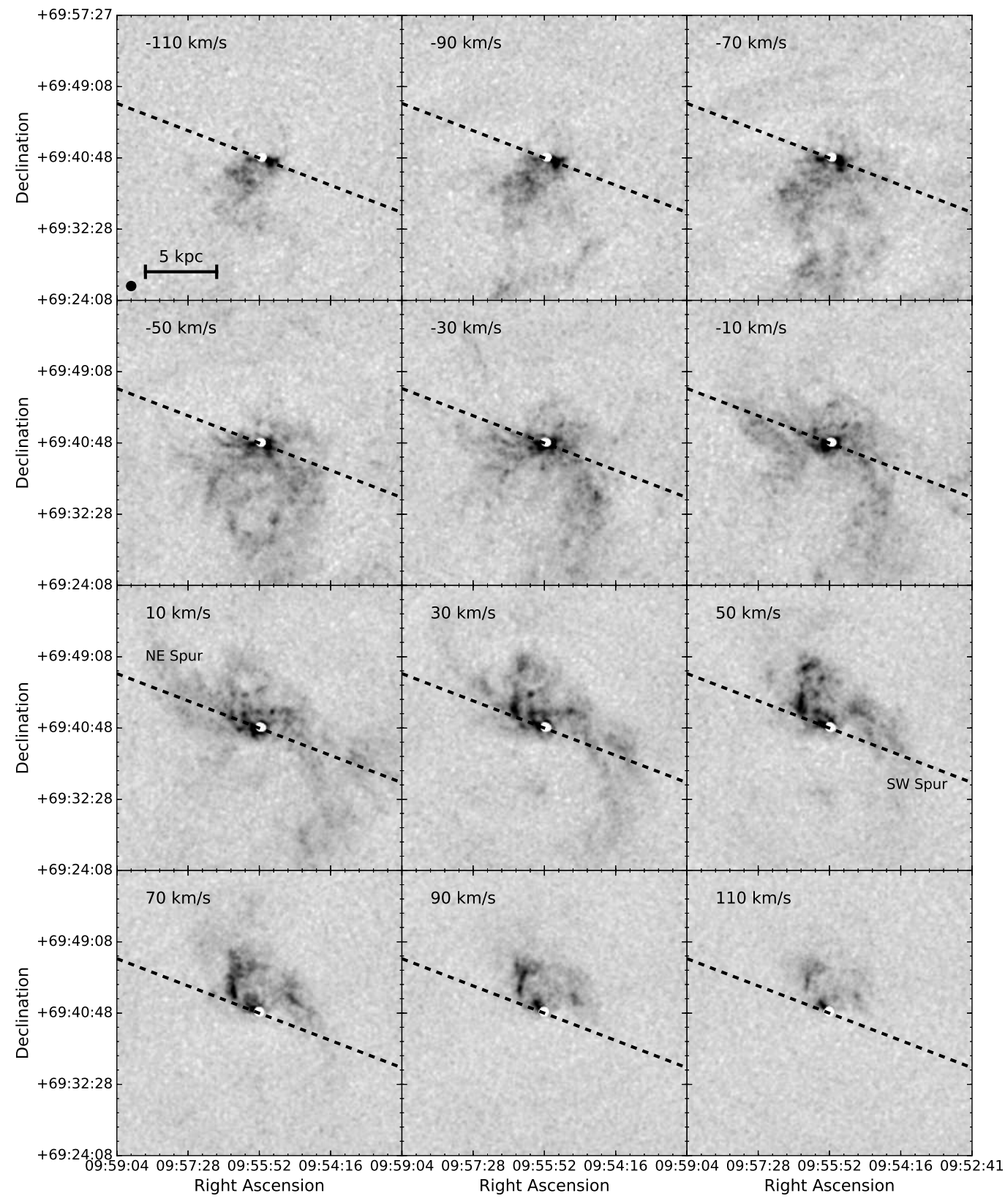

Figure 2. M82 H I intensity in twelve velocity channels after continuum subtraction. The central white area is due to $\mathrm{H}$ I absorption toward the center of the galaxy. The velocity for each channel is relative to the systemic velocity of M82. The major axis is oriented at $P A=67^{\circ}$ (black, dashed line). The projected size of $5 \mathrm{kpc}$ and the $24^{\prime \prime}$ FWHM beam diameter are shown in the upper left panel.

thin $\mathrm{H}$ I emission so that $N \approx 1.823 \times 10^{18} \mathrm{~cm}^{-2} I_{H I}$ with $I_{H I}$ in $\mathrm{K} \mathrm{km} \mathrm{s}^{-1}$. To highlight only bright, positive emission, we also created a mask that included all regions of the cube where the intensity exceeded a signalto-noise ratio of five over two successive channels. This corresponds to a column density limit of $\approx 7 \times 10^{19} \mathrm{~cm}^{2}$ across a $10 \mathrm{~km} \mathrm{~s}^{-1}$ velocity range. We used this mask to create integrated intensity and intensity-weighted mean velocity maps. Note that most of our analysis focuses on direct analysis of the cubes.

The integrated H I intensity map and velocity field are shown in Figure 1, and channel maps of $\mathrm{H}$ I intensity in select velocity channels are shown in Figure 2, The H I intensity map shows substantial H I extending along the major axis for over $\pm 5 \mathrm{kpc}$. The $\mathrm{H}$ I emission to the left (East) shows a significant spur that extends up (North) from the disk starting at about $4-5 \mathrm{kpc}$. This is the tidal stream seen in previous H I observations (Cottrell 1977; van der Hulst 1979; Appleton et al. 1981; Yun et al. 1993, 1994). This feature is labeled "NE Spur" in Figures 1]and 2, Our data also show some of the extensive pair of streams that extend west of the major axis, and then toward the South (Yun et al. 1993). This feature is labeled "SW Spur" in Figures 11 and 2. The $\mathrm{H}$ I emission along the minor axis extends approximately $5 \mathrm{kpc}$ toward the North and $10 \mathrm{kpc}$ toward the South. M82 is to the North of M81, so the southern part of M82 is closer to M81. 


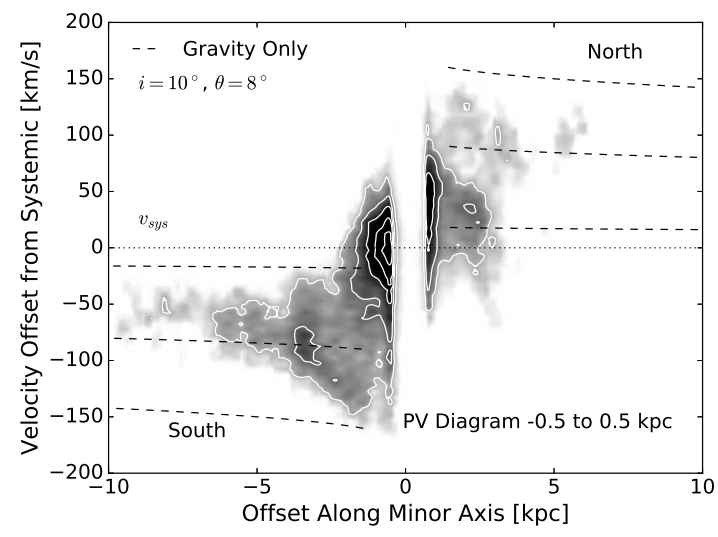

Figure 3. M82 minor axis position-velocity diagram. The grayscale and contours show the integrated H I intensity within $\pm 0.5 \mathrm{kpc}$ along the minor axis as a function of projected distance from the midplane. The velocity field has been shifted to the systemic velocity of $211 \mathrm{~km} \mathrm{~s}^{-1}$ (dotted line). $\mathrm{H} \mathrm{I}$ is clearly detected up to $10 \mathrm{kpc}$ to the South (left) and to about $6 \mathrm{kpc}$ to the North (right). Also shown are the ballistic velocities for test particles (dashed lines) for the mass model presented in 3 Three trajectories are shown in each direction. The central trajectory is aligned with the symmetry axis of a bicone tilted $\theta=10^{\circ}$ relative to the plane of the sky, while the other two trajectories are inclined by $\pm 8^{\circ}$ relative to the symmetry axis. This simple, ballistic model is not a good match to the data.

The H I velocity field shows some evidence of rotation, similar to the molecular gas (Young \& Scoville 1984; Yun et al. 1993; Leroy et al. 2015), although not as regular as the $K$-band stellar rotation curve measured by Greco et al. (2012). The H I rotation curve exhibits several drops in velocity (Sofue et al. 1992), which are most likely due to the influence of the bar (Telesco et al. 1991) and the tidal interaction (Yun et al. 1993). The velocity field along the minor axis has a steep gradient within about $1 \mathrm{kpc}$, where it decreases rapidly by over $100 \mathrm{~km} \mathrm{~s}^{-1}$ toward the South and increases rapidly by over $100 \mathrm{~km} \mathrm{~s}^{-1}$ toward the North. This spatial extent is where the start of the outflow is observed at other wavelengths (McKeith et al. 1995; Westmoquette et al. 2009). The amplitude of the velocity field then decreases toward the systemic velocity of M82. This is particularly prominent toward the South, where continuous, albeit filamentary, H I is detected out to $10 \mathrm{kpc}$ from the midplane. At this point the velocity has decreased to approximately half the value at $1 \mathrm{kpc}$. Figure 3 shows a position-velocity diagram along the minor axis within a synthesized slit of width $\pm 0.5 \mathrm{kpc}$ relative to the center of M82. The boundaries of this slit are shown on Figure 1]

\section{MASS MODEL}

We developed a mass model for M82 based on measurements of the stellar surface photometry, stellar kinematics, and simulations of its interaction history within the M81 group. This model includes a bulge, a disk, and a dark matter halo and was constructed with the galpy software package (Bovy 2015). We took the physical length scale of the bulge and disk components from the wide-field, near-infrared surface photometry of Ichikawa et al. (1995). They performed a bulge-disk decomponsition and found the bulge light along the major axis is well fit by a de Vaucouleurs $R^{1 / 4}$ law with an effective radius of $21^{\prime \prime}\left(K^{\prime}\right.$-band) to $29^{\prime \prime}(H$-band $)$.

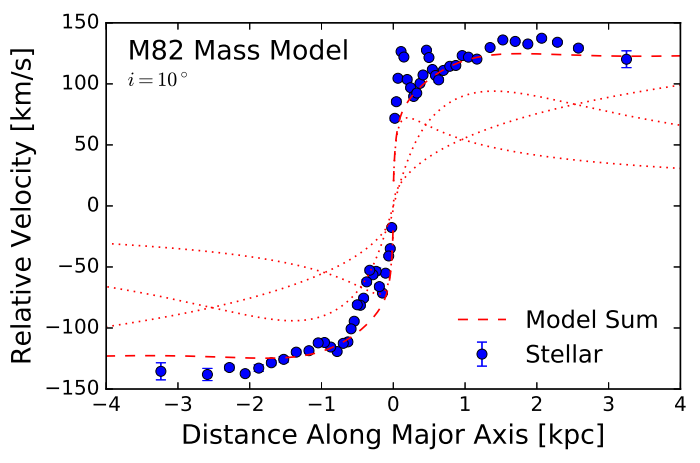

Figure 4. M82 rotation curve model and observations. The data points (with error bars) are the stellar kinematics measured by Greco et al. (2012) with the $2.29 \mu \mathrm{m}$ CO bandhead in the nearinfrared $K$-band. The lines show the rotation curve (dashed) and three individual components (dotted) calculated from the mass model described in $\$ 3$ The bulge component is the most significant component near the center of the galaxy, the disk dominates out to several $\mathrm{kpc}$, and the halo at larger radii.

We adopt a physical radius of $r_{e}=0.36 \mathrm{kpc}$ for this component, which we model with a Hernquist potential (Hernquist 1990) with $a=0.2 \mathrm{kpc}$ (with the relation $\left.a=r_{e} / 1.8153\right)$. Ichikawa et al. (1995) measured an exponential disk scale length of $40^{\prime \prime}$, and we adopt a physical disk of $r_{s}=0.6 \mathrm{kpc}$. We model the exponential disk with the galpy implementation of the Smith et al. (2015) prescription to describe an exponential disk as three Miyamoto-Nagai disks (Mivamoto \& Nagai 1975). We set the vertical scale height to $h_{z}=0.1 \mathrm{kpc}$. This quantity was not calculated by Ichikawa et al. (1995), but the exact choice does not appreciably impact the kinematics above the midplane. Lastly, we model the dark matter halo as a Navarro et al. (1996, NFW) profile. H I observations of the M81 group show long tidal features that are good evidence of past interactions between M81 and M82, as well as between M81 and NGC 3077 (Cottrell 1977; Yun et al. 1993). Oehm et al. (2017) performed a thorough simulation study of various scenarios for the interaction history of the M81 group and placed constraints on the dark matter profiles of the largest members. We adopt their NFW parameters of $R_{200}=164$ kpc, $\rho_{0}=8.81 \times 10^{-3} \mathrm{M}_{\odot} \mathrm{pc}^{-3}$, and $c=11.17$.

There are two free parameters in the mass model: the masses of the bulge and disk components. We adjust these parameters until we obtain a reasonably good match to the major axis rotation curve measured by Greco et al. (2012). That rotation curve was measured from the CO $2.29 \mu \mathrm{m}$ bandhead that is prominent in the near-infrared spectra of giant and supergiant stars. This rotation curve is shown in Figure 4, along with the rotation curves of the three mass components. The mass we adopt for the bulge component is $2 \times 10^{9} \mathrm{M}_{\odot}$, although we note that much of this mass may not be in the form of the old stellar population characteristic of classical bulges. One reason is that there is good evidence from stellar spectroscopy that the central light distribution is dominated by young supergiants (Rieke et al. 1993; Förster Schreiber et al. 2001, 2003; Greco et al. 2012), rather than the old stellar population typical of classical bulges. A substantial fraction of the mass in the central region is also in the form of atomic and molecular gas (Young \& Scoville 1984; Walter et al. 
2002; Leroy et al. 2015). Regardless of the exact form of the mass, this component dominates the rotation curve in the central $0.5 \mathrm{kpc}$. We normalize the disk component to $8 \times 10^{9} \mathrm{M}_{\odot} \mathrm{kpc}^{-3}$, and this is the most prominent component out to about $2 \mathrm{kpc}$. The total mass of our model is in good agreement with the total dynamical mass of $\sim 10^{10} M_{\odot}$ derived by Greco et al. (2012), and the range of $10^{9-10} M_{\odot}$ derived by previous studies, although those studies analyzed a smaller range of radii (Young \& Scoville 1984; Goetz et al. 1990; Sofue et al. 1992).

\section{ANALYSIS}

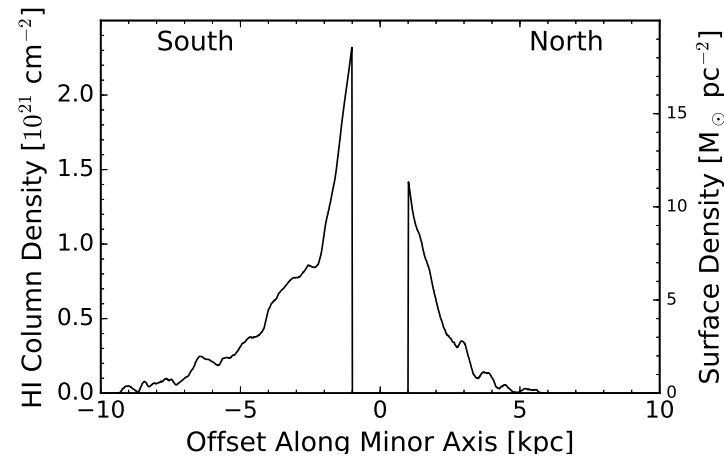

Figure 5. HI column density vs. distance from the midplane in the same $\pm 0.5 \mathrm{kpc}$ region used to construct Figures [3 and 6] The central $\pm 1 \mathrm{kpc}$ is masked as there is significant $\mathrm{H}$ I absorption near the midplane. The right axis shows the equivalent surface density of neutral Hydrogen calculated from $8 \times 10^{-21} N_{H}$.

We detect substantial H I emission along the minor axis out to at least $5 \mathrm{kpc}$ to the North and $10 \mathrm{kpc}$ to the South (Figure 1). The total H I intensity is also greater toward the South than toward the North, and there is more molecular gas and dust to the South (Walter et al. 2002; Lerov et al. 2015). Figure 5 shows the H I column density as a function of distance from the midplane along the minor axis in the same $\pm 0.5 \mathrm{kpc}$ region used to construct Figure 3

The spatial distribution and kinematics of the $\mathrm{H}$ I gas are a poor match to the outflow traced by the hottest gas. Strickland \& Stevens (2000) discuss how the hot wind fluid traced by their X-ray observations may accelerate cold clouds by ram pressure. Strickland \& Heckman (2009) estimate the terminal velocity of the wind is $v_{\infty}=1410-2240 \mathrm{~km} \mathrm{~s}^{-1}$, which is greater than the escape velocity of $v_{\text {esc }}=460 \mathrm{~km} \mathrm{~s}^{-1}$ for M82. The minor axis kinematics of the warm, ionized gas traced by $\mathrm{H} \alpha$ and other visible-wavelength lines suggest velocities of $500-600 \mathrm{~km} \mathrm{~s}^{-1}$ for an $80^{\circ}$ disk inclination (McKeith et al. 1995; Shopbell \& Bland-Hawthorn 1998). The velocity of the warm ionized gas is consequently substantially lower than the hot wind fluid, and closer to M82's escape velocity (Heckman et al. 2000). The H I kinematics are similar to the warm, ionized gas, as well as the colder, molecular gas traced by $\mathrm{CO}$ (Walter et al. 2002; Lerov et al. 2015). The similar kinematics supports the hypothesis that the warm ionized gas and the cooler atomic and molecular gas are cospatial, but they are not cospatial with the hot wind fluid detected at X-ray energies.
Further evidence that the warm ionized gas and cooler atomic and molecular gas are cospatial comes from measurements of line splitting along the minor axis that suggests both gas phases trace the edge of a bicone that surrounds the higher temperature X-ray fluid. This evidence includes the Fabry-Perot observations of Bland \& Tully (1988) and line splitting in longslit spectroscopy. Heckman et al. (1990) used longslit spectroscopy to derive an opening angle of $60^{\circ}$ (and angles of $30-40^{\circ}$ between the symmetry axis of the cone and the plane of the sky). McKeith et al. (1995) derived a smaller cone opening angle of $30^{\circ}$ at $>1 \mathrm{kpc}$. Line splitting is also observed in the colder gas traced by $\mathrm{H} \mathrm{I}$ and CO. Leroy et al. (2015) derived a smaller cone opening angle of $13-20^{\circ}$ for this cold gas. The velocity spread of the minor axis $\mathrm{H}$ I observed in our new observations is more consistent with the smaller opening angle derived by Lerov et al. (2015). A possible explanation for the discrepancy between the measurements from warm, ionized gas and colder atomic gas are that the later measurements were made further from the midplane, where the outflow may be more columnated.

\subsection{Minor Axis Velocity Decrease}

The most remarkable feature of the minor axis $\mathrm{H} \mathrm{I}$ kinematics is the decrease of the typical velocity amplitude from about $100 \mathrm{~km} \mathrm{~s}^{-1}$ to $50 \mathrm{~km} \mathrm{~s}^{-1}$ over the projected distance range from about $1.5 \mathrm{kpc}$ to $10 \mathrm{kpc}$ (Figure 31). This decline is very obvious along the minor axis toward the South. The kinematics to the North are broadly consistent with this same velocity profile, although the $\mathrm{H}$ I intensity is substantially less and the gas is only detected to about $5 \mathrm{kpc}$ in projection.

The velocity amplitude decrease from 1.5 to $10 \mathrm{kpc}$ is not consistent with $\mathrm{H}$ I clouds that have been accelerated by the wind and then launched on ballistic trajectories, and consequently demonstrate that an additional force is required. This is because the inferred outflow velocities of these clouds is on order $500-600 \mathrm{~km} \mathrm{~s}^{-1}$ (for $80^{\circ}$ disk inclination) at a projected height of $1.5 \mathrm{kpc}$ and the mass of M82 is insufficient to slow the clouds by $50 \%$ from 1.5 $\mathrm{kpc}$ to $10 \mathrm{kpc}$. This point is illustrated by the dashed lines in Figure 3. The lines show the projected velocity of a test particle calculated with galpy and the mass model for M82 developed in \$3. The middle line shows the trajectory for a test particle on the symmetry axis of the bicone (at an angle of $10^{\circ}$ relative to the plane of the sky) and the other lines show test particles at $\pm 8^{\circ}$ on the nominal edges of a cone with a $16^{\circ}$ opening angle. The H I kinematics clearly decrease much faster than expected from gravitational forces alone. They are also inconsistent with cooling of the $\mathrm{H}$ I gas from the hot wind. $\mathrm{H}$ I produced from cooling in a hot wind should have the velocity of the hot wind, which Strickland \& Heckman (2009) estimate to be as high as $\sim 2000 \mathrm{~km} \mathrm{~s}^{-1}$. The $\mathrm{H}$ I kinematics also demonstrate that the cold $\mathrm{H}$ I clouds do not experience continual acceleration, nor even free expansion into the halo of M82.

One possible explanation for this decrease in projected velocity is that the inclination of the bicone is substantially different from the stellar disk, as this would decrease the $\mathrm{H}$ I outflow velocity. We find that the $\mathrm{H}$ I velocities do diminish by the required amount from $1.5-5$ kpc if the symmetry axis of the cone is tilted toward us by 


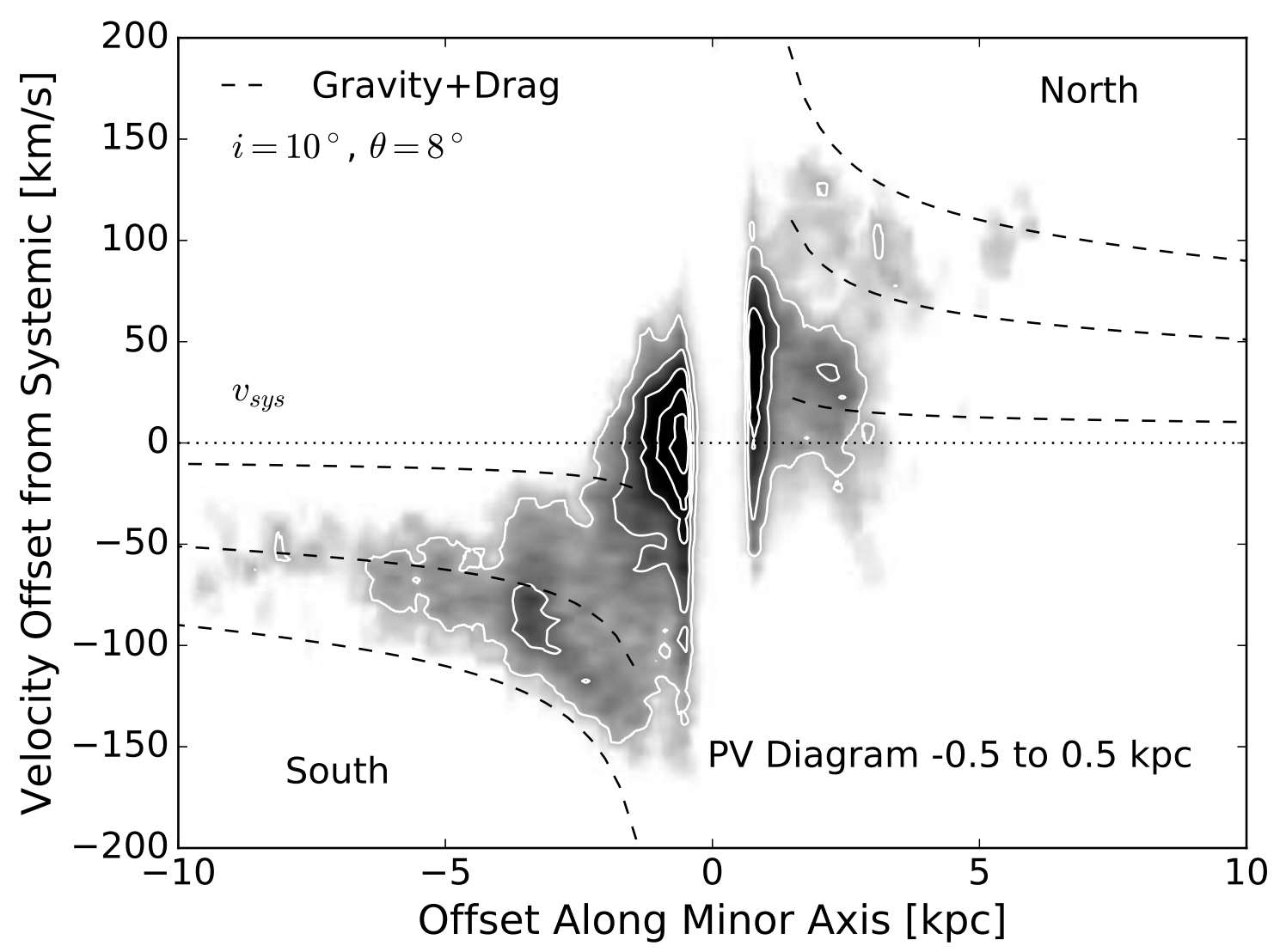

Figure 6. Same as Figure 3 except with a model that includes both gravitational and drag forces on the H I gas.

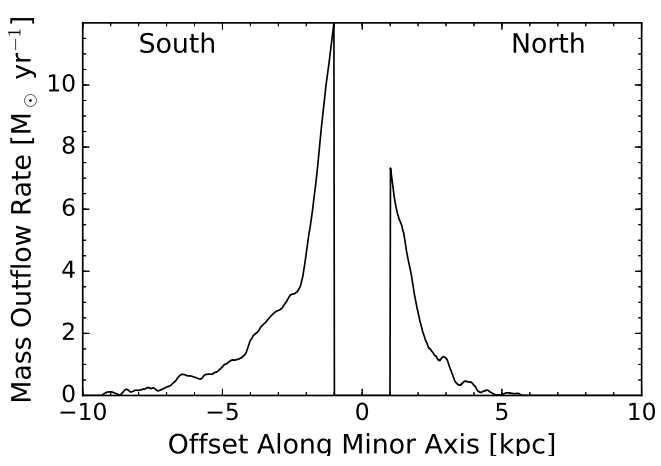

Figure 7. Integrated mass outflow rate within $\pm 0.5 \mathrm{kpc}$ of the minor axis derived from the surface density shown in Figure 5 and the velocity profile of the best-fit model. The central $\pm 1 \mathrm{kpc}$ is masked as there is significant $\mathrm{H} \mathrm{I}$ absorption near the midplane.

$30^{\circ}$ relative to the plane of the sky. However, in this case the $\mathrm{H}$ I velocities continue to decrease to the systemic velocity, and do not reproduce the relatively flat velocity profile seen from $5-10 \mathrm{kpc}$. Other possible explanations include that the $\mathrm{H}$ I experiences some additional drag force, the $\mathrm{H}$ I does not represent a continuous stream of material, but instead is forming in situ (although not from the hot wind), and that the $\mathrm{H}$ I kinematics have been substantially affected by tidal interactions.

If the $\mathrm{H}$ I consists of clouds flowing outward along the edge of the bicone, they could experience a drag force if they encounter ambient material outside of the bicone. This material could be halo gas of M82 that is not part of the wind, or tidal material that is approx- imately stationary relative to the wind. We model the drag force with the drag equation $f_{d r a g}=C_{d} \rho_{a m b} v^{2} A$, where $C_{d}$ is the drag coefficient, $\rho_{a m b}$ is the density of the ambient medium, $v$ is the velocity of the $\mathrm{H}$ I relative to this medium, and $A$ is the cross section of a typical H I cloud. We then make the assumption that $C_{d} \rho_{a m b} A \propto r^{-2}$. This would be true if $C_{d} A$ were constant and $\rho_{a m b} \propto r^{-2}$. The coefficient $C_{d}$ is approximately constant for spheres (of constant cross section A) in a supersonic flow, while $\rho_{a m b} \propto r^{-2}$ is characteristic of mass outflow at a constant rate at constant velocity. Based on measurements by Leroy et al. (2015), we expect the density profile is somewhat steeper with $\rho_{a m b} \propto r^{-3 \rightarrow-4}$ for the dust and molecular gas 8 . To maintain our assumption in this case would require the drag coefficient to increase with $r$, which could happen if the Reynolds number decreased with radius. We find that the addition of a drag force with $C_{d} \rho_{a m b} A \propto r^{-2}$ provides a reasonable match to the data. This model is shown in Figure 6.

If the velocity change of the $\mathrm{H} I$ gas is due to a drag force imposed by the ambient medium, the nongravitational deceleration of the test particles that best match the data constrain the product of $C_{d} \rho_{a m b} A_{c l}$. If we assume these are spherical clouds of $\mathrm{H}$ I gas with uniform density $\rho_{c l}$ and radius $R_{c l}$, the non-gravitational

8 While Lerov et al. (2015) did measure $r^{-2}$ for $\mathrm{H}$ I, that is more due to the abundance of tidal material, than because the wind material follows $r^{-2}$. 
deceleration is:

$$
\frac{d v}{d t}=\frac{3}{4} C_{d}\left(\frac{\rho_{a m b}}{\rho_{c l}}\right) \frac{v_{c l}^{2}}{R_{c l}}
$$

At $2 \mathrm{kpc}$ from the midplane, the deprojected $\mathrm{H}$ I velocity is about $500 \mathrm{~km} \mathrm{~s}^{-1}$ and the best-fit model has a non-gravitational deceleration of $1.8 \times 10^{-7} \mathrm{~cm} \mathrm{~s}^{-2}$. For $R_{c l}=10 \mathrm{pc}$, the density ratio is $\sim 0.0044\left(0.5 / C_{d}\right)$. If the cloud particle density is $10 \mathrm{~cm}^{-3}$, the ambient medium at $2 \mathrm{kpc}$ from the midplane must be relatively dense with $0.044 \mathrm{~cm}^{-3}$. This is substantially denser than the hot wind, although the wind has much larger velocities so it is unlikely to be the same medium. If drag forces decelerate the $\mathrm{H}$ I gas, then the ambient medium responsible for the deceleration must be distinct from the hot wind, and at higher density than the typical hot, coronal gas of galactic halos. This could be due to material stripped out of the disk by the tidal interaction with M81. The tidal interaction may also be the main origin of the decrease in the $\mathrm{H}$ I velocity, rather than a drag force due to the ambient medium.

The combination $\rho_{c l} R_{c l}$ in Equation 1 is the column density of the clouds in the outflow. If the majority of the material in the cloud is $\mathrm{H} \mathrm{I}$, then this quantity can be measured with $\mathrm{H}$ I emission maps or UV/visible absorption line studies with sufficiently high resolution and sensitivity. Such a measurement, combined with the acceleration and the velocity of the clouds, could then be used to solve for the ambient density required to produce the observed deceleration. The angular size of a $\sim 10-20 \mathrm{pc}$ cloud at the distance of M82 is $0.5-1^{\prime \prime}$. This is a challenge for current $\mathrm{H}$ I facilities, but may be feasible with $\mathrm{UV} /$ visible absorption line studies toward background sources.

Similar to Leroy et al. (2015), we have used the mass distribution and velocity information to derive the mass flux associated with the H I emission. Under the assumption that all of the $\mathrm{H}$ I is associated with an outflow, Figure 7 shows the mass flux computed from the product of the mass surface density (Figure 5), the velocity from our outflow model, and the $\pm 0.5 \mathrm{kpc}$ width of the central region of our position-velocity diagram. The main differences from the calculation and figure shown in Leroy et al. (2015) are that we used a smaller region width (1 kpc, rather than $3 \mathrm{kpc})$ and we used the velocity profile, rather than a fixed outflow speed of $450 \mathrm{~km} \mathrm{~s}^{-1}$. While the mass flux we have derived near the midplane is lower than in Leroy et al. (2015), largely because of the smaller region width, the mass flux is detected out to about twice the distance from the midplane, although it drops to a fraction of a solar mass per year beyond $4 \mathrm{kpc}$. This is also approximately the point where the mass flux is comparable to the outflow rate computed by Strickland \& Heckman (2009) for the hot gas. This H I outflow rate is about an order of magnitude less than the star formation rate.

The H I velocity decrease along the minor axis may be so substantial that the $\mathrm{H}$ I will not escape the halo. At a projected height of $10 \mathrm{kpc}$, the line of sight velocity has dropped to $50 \mathrm{~km} \mathrm{~s}^{-1}$ relative to the systemic velocity, which corresponds to an inclination-corrected outflow velocity of about $300 \mathrm{~km} \mathrm{~s}^{-1}$. This is sufficiently comparable to the escape velocity of our mass model that the ultimate fate of the gas will depend on assumptions in our model for the drag force on the cold gas, the impact of tidal forces in the M81 group, and the halo model for M82. Within the context of our model, the H I would finally stall at $70-80 \mathrm{kpc}$ after about $500 \mathrm{Myr}$. The H I gas could consequently form a cold "fountain" as proposed by Leroy et al. (2015), rather than substantially add to the intergalactic medium. We searched in our data for any evidence of material that is falling back, but do not see any. This included a careful inspection of the non-masked data, which has somewhat greater sensitivity, although at the expense of more artifacts.

\subsection{Biconical Frustum}

A simple bicone model is not a good match to the spatial and velocity distribution of the $\mathrm{H}$ I gas. Figures 1 and 2 clearly show the H I gas does not have a conical shape. The opening angle derived from line splitting in the cold gas by Lerov et al. (2015) is also smaller than values derived closer to the plane from the warm, ionized gas (Heckman et al. 1990; McKeith et al. 1995), which suggests the cold gas is more columnated. Another argument against a simple bicone is the presence of outflowing material at offset positions parallel to the minor axis. Figure 1 shows five slices parallel to the minor axis that are $1 \mathrm{kpc}$ wide and offset at $+2,+1,0,-1$, and -2 kpc along the midplane. Figure 8 shows the position velocity at each of those five positions. This figure clearly shows the $H$ I velocity profile observed within $\pm 0.5 \mathrm{kpc}$ of the minor axis (Center) is also seen in positions offset by as much as $\pm 2 \mathrm{kpc}$, albeit at somewhat lower intensity. A simple bicone with an opening angle of $8^{\circ}$ (half width) would have no flux within about $\pm 7 \mathrm{kpc}$ of the major axis in the offset positions at $\pm 2 \mathrm{kpc}$. While the intensity at these offset positions could be explained with a larger opening angle, a larger opening angle would also produce a much larger range of velocities at each position that would not be consistent with the observations.

We have consequently compared the data to a biconical frustum, rather than a simple bicone. Since a frustum is a conical shape with the top removed, we use this representation to model the origin of the outflow from a broader region of the disk than the single point of a cone. This shape is a better physical match to the spatial extent of the starburst, yet maintains the relatively narrow range of velocities observed further from the midplane (see Westmoquette et al. 2011, for the application of a frustum to NGC 253). We find that a frustum radius of $1 \mathrm{kpc}$ at the midplane and an opening angle half width of $8^{\circ}$ is a reasonable match to the data. The outline of this biconical frustum is shown in Figure 1, and position-velocity diagrams for this model are shown on the right side of Figure 8 at the same five offset positions as the data on the left. The choice of a $1 \mathrm{kpc}$ radius at the midplane was motivated by the physical extent of the starburst region $(\sim 500$ pc Förster Schreiber et al. 2003), but increased to provide a better match to the observed position-velocity diagrams. The velocity of the gas in the model was calculated at each point based on the radial distance from the center of the model. The velocity includes gravity and drag forces as in Figure 6. and was normalized such that the initial velocity of the gas matches the observed $\mathrm{H}$ I kinematics at $1.5 \mathrm{kpc}$ from the midplane. The model does not include any material 

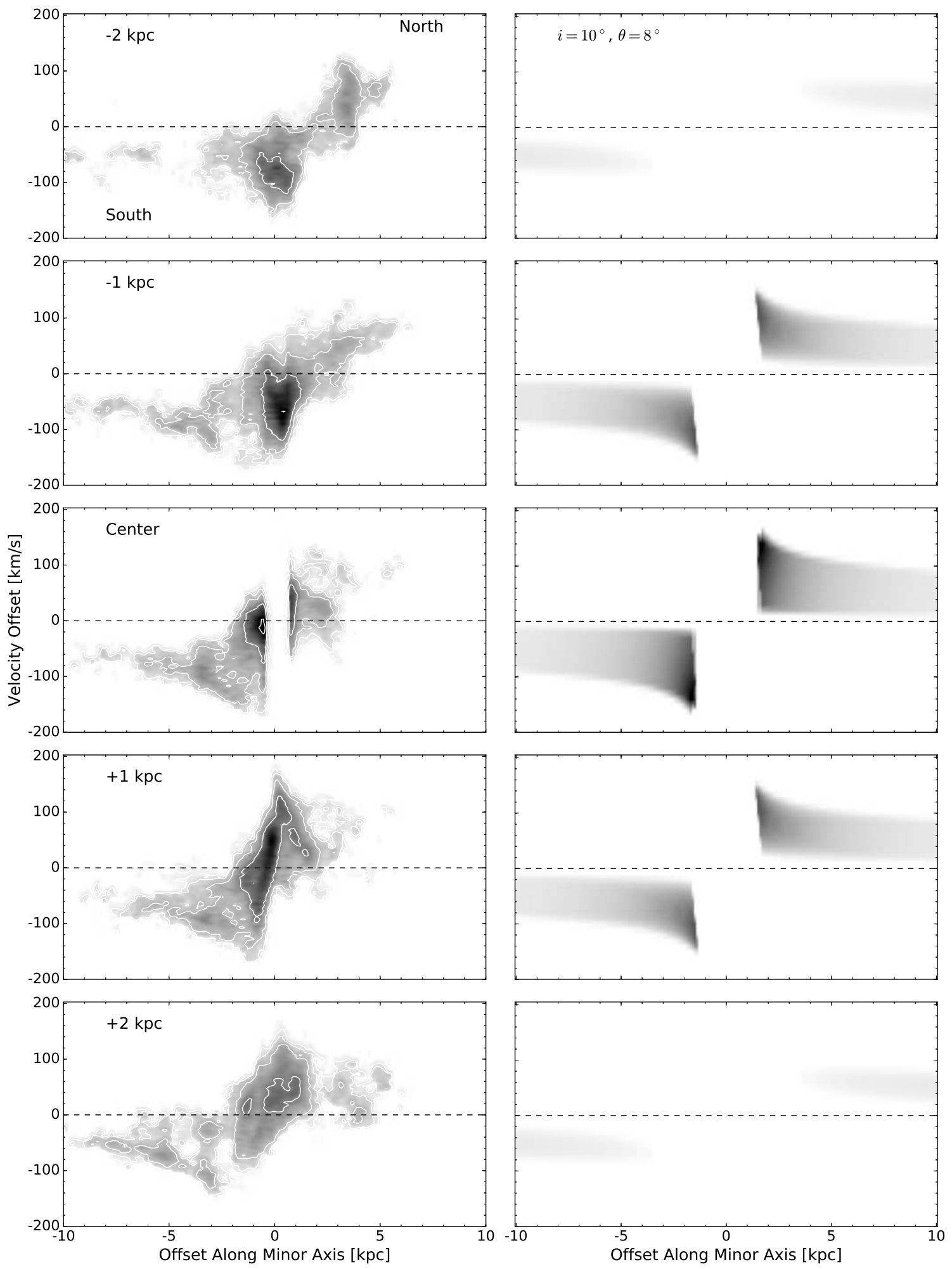

Figure 8. Five position-velocity diagrams offset for the observed H I gas (left) and a model biconical frustum outflow with an $\mathrm{R}=1 \mathrm{kpc}$ base on the major axis (right). Each row shows a $1 \mathrm{kpc}$ wide slice perpendicular to the major axis. The H I data show the five positions marked in Figure 1 while the model shows the projected position-velocity diagram of a model filled biconical frustum with $i=10^{\circ}$ and opening angle $\theta=8^{\circ}$ (half width). The model includes both the gravitational deceleration and the drag force shown in Figure 6 . 
closer than $1.5 \mathrm{kpc}$ from the center, as we assumed this region is substantially affected by the disk kinematics. The main disagreement between the model and data are that the model has a somewhat broader velocity distribution. This could be because our simple model adopts a uniformly filled frustum. The model would better match the data if the material were more concentrated on the symmetry axis, although physically that would be in conflict with the expected location of the hot wind. At larger distances, the density is assumed to decrease as $n \propto r^{-2}$.

\subsection{Alternative Scenarios}

It is also possible that the H I gas does not represent a continuous population of clouds flowing from the disk, but is instead partially or completely produced in situ. In this case, one potential origin is the dissociation of molecular gas. The molecular gas measurements from Lerov et al. (2015) within a few kpc of the midplane show similar kinematics as the atomic gas; however, the mass density of molecular gas is substantially lower than the $\mathrm{H}$ I off the midplane, and decreases much more rapidly with distance from M82. Deeper molecular gas observations would help to further test this hypothesis, along with calculations of the disassociation rate.

Another possibility is that the $\mathrm{H}$ I gas has radiatively cooled from the hot phase (Wang 1995a, b). The basic physical picture, which was developed in detail by Thompson et al. (2016), starts with cool gas clouds in the disk that are initially accelerated and shredded by the hot wind. The addition of this cloud material then increases the mass loading in the wind, and then this additional mass seeds thermal and radiative instabilities that precipitate cool gas out in the wind at larger distances. Following the wind model of Chevalier \& Clegg (1985), a key dimensionless parameter is the mass loading parameter $\beta$, which describes the ratio of the mass outflow rate $\dot{M}_{W}$ in the wind to the star formation rate $\dot{M}_{*}$, that is $\dot{M}_{W}=\beta \dot{M}_{*}$. The radiative cooling requires some minimum mass loading due to the shape of the cooling function. For the case of M82, Hoopes et al. (2003) find weak evidence of cooling on large scales and Strickland \& Heckman (2009) estimate a fairly low mass loading factor of $\beta=0.2-0.5$ in the core, although the wind could be strongly mass loaded on larger scales. Thompson et al. (2016) therefore conclude that their model may not apply to M82, and note the additional complication of tidal material. The monotonic decline in the characteristic $\mathrm{H}$ I velocity with projected distance also does not seem consistent with radiative cooling because the velocity profile is inconsistent with the inferred velocity profile for the hot wind.

There is some phenomenological similarity between the velocity decrease in M82 on kpc scales and the ionized gas kinematics observed in nearby AGN on scales approximately an order of magnitude smaller. Studies of Seyfert galaxies such as NGC 1068 (Crenshaw \& Kraemer 2000), Mrk 3 (Ruiz et al. 2001), and NGC 4151 (Das et al. 2005) are reasonably well matched with constant acceleration to $100-300 \mathrm{pc}$, followed by constant deceleration to the systemic velocity. Everett \& Murrav (2007) explored Parker wind models to explain the $\sim 100$ pc outflows in AGN, but found no self-consistent temperature profile that could explain all of the kinematics. They did note that the generic hydromagnetic winds explored by Matzner \& McKee (1999) could explain the acceleration, and there are morphological similarities between that model and the observations of Mav \& Steiner (2017), but it was not clear how to explain the deceleration without interaction with the surrounding medium.

\section{SUMMARY}

We have presented new VLA observations of the archetypical starburst galaxy M82, and combined them with earlier observations from the GBT. These data trace $\mathrm{H}$ I emission out to a projected distance of $10 \mathrm{kpc}$ from the center of the galaxy with an angular resolution of $\approx 24^{\prime \prime}$. The velocity amplitude to both the North and South decreases from about $140 \rightarrow 50 \mathrm{~km} \mathrm{~s}^{-1}$ over a projected distance of $1.5 \rightarrow 5 \mathrm{kpc}$ from the midplane. The $\mathrm{H}$ I intensity is substantially greater to the South, where $\mathrm{H} \mathrm{I}$ is detected out to a projected distance of $10 \mathrm{kpc}$.

These minor axis H I kinematics are not consistent with continual acceleration of the atomic gas from the midplane, nor with gas clouds launched on approximately ballistic trajectories. The H I gas is also three to four times slower than the inferred velocity of the hot superwind fluid traced by X-ray data, and therefore the $\mathrm{H}$ I gas is not consistent with material that has cooled from that phase. The H I kinematics are more similar to the molecular and warm, ionized gas that appears to trace the outer sheath of the superwind, although the warm, ionized gas has only been measured closer to the midplane.

We use a new mass model for M82 to demonstrate that an additional force is required to slow the $\mathrm{H}$ I gas, and that the observed velocity profile is consistent with drag caused by the ambient environment. This requires that the H I gas is spatially distinct from the hot superwind fluid, and is not just the cool tail of the temperature distribution. The magnitude of the required drag force constrains the density ratio of the clouds and ambient medium, as well as the typical cloud size, which could be tested with future, higher resolution H I observations. The high velocities and density contrast between the outflow and ambient medium would also produce shockheated gas, which may contribute to the X-ray emission.

One alternate possibility is that the $\mathrm{H}$ I gas is not a continuous stream of material flowing from the disk, but rather is partially or completely produced in situ by dissociation of the molecular gas and/or adiabatic cooling of the warm, ionized gas that forms the interface between the superwind and the ambient medium. We disfavor this scenario because it does not naturally explain the systematic decrease in the $\mathrm{H}$ I velocities with distance from the midplane, at least not without a similar drag force on that material. There is also a very substantial velocity contrast between the superwind and the other phases. Deeper observations of the molecular and especially the warm ionized gas that extend further from the midplane would help reveal the extent to which that material shares the same kinematic profile as the $\mathrm{H}$ I gas.

A final possibility is that the $\mathrm{H}$ I along the minor axis is largely tidal debris viewed in projection near the minor axis. Such a superposition would be an unfortunate coincidence, and does not appear consistent with either the symmetric kinematic profile about the midplane or the larger-scale tidal streams of H I. Deeper, wider-field 
H I observations, combined with new models of the interactions in the M81 group, could help to quantify the contribution of $\mathrm{H}$ I tidal streams, if any, to the $\mathrm{H}$ I observed along the minor axis.

The ultimate fate of the H I gas is unclear. Our detection of the velocity decrease along the minor axis, combined with our new mass model for M82, demonstrates that there is likely some additional drag force that slows the cold phase of the wind. This velocity decrease lowers the wind speed to be comparable to the escape velocity from the halo, and thus the ultimate fate of the gas could be fallback onto the disk in a cold fountain on $\sim 100 \mathrm{Myr}$ timescales, long-term residence in the circumgalactic medium, or escape into the intergalactic medium. How the gas is distributed among these three scenarios depends on any additional drag forces on the wind at larger distances, details of the halo mass distribution, and the tidal field of the M81 group.

The National Radio Astronomy Observatory is a facility of the National Science Foundation operated under cooperative agreement by Associated Universities, Inc. We thank Todd Thompson for discussions of Galactic winds and outflows. We also thank Norm Murray for his helpful referee report. The work of PM is partially supported by the National Science Foundation under Grant 1615553 and by the Department of Energy under Grant de-sc0015525. The work of AKL is partially supported by the National Science Foundation under Grants No. 1615105, 1615109, and 1653300. ADB acknowledges support from the National Science Foundation grant AST1412419.

Facilities: VLA, GBT.

\section{REFERENCES}

Amirkhanyan, A. S., Gagen-Torn, V. A., \& Reshetnikov, V. P. 1982, Astrophysics, 18, 17

Andrews, B. H., \& Thompson, T. A. 2011, ApJ, 727, 97

Appleton, P. N., Davies, R. D., \& Stephenson, R. J. 1981, MNRAS, 195, 327

Axon, D. J., \& Taylor, K. 1978, Nature, 274, 37

Bland, J., \& Tully, B. 1988, Nature, 334, 43

Borthakur, S., Heckman, T., Strickland, D., Wild, V., \& Schiminovich, D. 2013, ApJ, 768, 18

Bovy, J. 2015, ApJS, 216, 29

Chevalier, R. A., \& Clegg, A. W. 1985, Nature, 317, 44

Chynoweth, K. M., Langston, G. I., Yun, M. S., et al. 2008, AJ, 135,1983

Chynoweth, K. M., Langston, G. I., Holley-Bockelmann, K., \& Lockman, F. J. 2009, AJ, 138, 287

Chynoweth, K. M., Langston, G. I., \& Holley-Bockelmann, K. 2011, AJ, 141, 9

Coker, C. T., Thompson, T. A., \& Martini, P. 2013, ApJ, 778, 79

Cottrell, G. A. 1977, MNRAS, 178, 577

Crenshaw, D. M., \& Kraemer, S. B. 2000, ApJL, 532, L101

Das, V., Crenshaw, D. M., Hutchings, J. B., et al. 2005, AJ, 130, 945

Efstathiou, G. 2000, MNRAS, 317, 697

Engelbracht, C. W., Kundurthy, P., Gordon, K. D., et al. 2006, ApJL, 642, L127

Everett, J. E., \& Murray, N. 2007, ApJ, 656, 93

Finlator, K., \& Davé, R. 2008, MNRAS, 385, 2181

Freedman, W. L., Hughes, S. M., Madore, B. F., et al. 1994, ApJ, 427,628

Förster Schreiber, N. M., Genzel, R., Lutz, D., Kunze, D., \& Sternberg, A. 2001, ApJ, 552, 544

Förster Schreiber, N. M., Genzel, R., Lutz, D., \& Sternberg, A. 2003, ApJ, 599, 193
Gerke, J. R., Kochanek, C. S., Prieto, J. L., Stanek, K. Z., \& Macri, L. M. 2011, ApJ, 743, 176

Goetz, M., Downes, D., Greve, A., \& McKeith, C. D. 1990, A\&A, 240,52

Greco, J. P., Martini, P., \& Thompson, T. A. 2012, ApJ, 757, 24

Heckman, T. M., Armus, L., \& Miley, G. K. 1990, ApJS, 74, 833

Heckman, T. M., Lehnert, M. D., Strickland, D. K., \& Armus, L. 2000, ApJS, 129, 493

Hernquist, L. 1990, ApJ, 356, 359

Hoopes, C. G., Heckman, T. M., Strickland, D. K., \& Howk, J. C. 2003, ApJL, 596, L175

Hunter, D. A., Ficut-Vicas, D., Ashley, T., et al. 2012, AJ, 144, 134

Ichikawa, T., Yanagisawa, K., Itoh, N., et al. 1995, AJ, 109, 2038

Langston, G., \& Turner, B. 2007, ApJ, 658, 455

Leroy, A. K., Walter, F., Martini, P., et al. 2015, ApJ, 814, 83

Lynds, C. R., \& Sandage, A. R. 1963, ApJ, 137, 1005

Matzner, C. D., \& McKee, C. F. 1999, ApJL, 526, L109

May, D., \& Steiner, J. E. 2017, MNRAS, 469, 994

McCourt, M., O'Leary, R. M., Madigan, A.-M., \& Quataert, E. 2015, MNRAS, 449, 2

McKeith, C. D., Castles, J., Greve, A., \& Downes, D. 1993, A\&A, 272,98

McKeith, C. D., Greve, A., Downes, D., \& Prada, F. 1995, A\&A, 293, 703

Miyamoto, M., \& Nagai, R. 1975, PASJ, 27, 533

Murray, N., Quataert, E., \& Thompson, T. A. 2005, ApJ, 618, 569

Murray, N., Quataert, E., \& Thompson, T. A. 2010, ApJ, 709, 191

Navarro, J. F., Frenk, C. S., \& White, S. D. M. 1996, ApJ, 462, 563

Oppenheimer, B. D., \& Davé, R. 2008, MNRAS, 387, 577

Oehm, W., Thies, I., \& Kroupa, P. 2017, MNRAS, 467, 273

Ott, J., Stilp, A. M., Warren, S. R., et al. 2012, AJ, 144, 123

Peeples, M. S., \& Shankar, F. 2011, MNRAS, 417, 2962

Rieke, G. H., Loken, K., Rieke, M. J., \& Tamblyn, P. 1993, ApJ, 412, 99

Roussel, H., Wilson, C. D., Vigroux, L., et al. 2010, A\&A, 518, L66

Ruiz, J. R., Crenshaw, D. M., Kraemer, S. B., et al. 2001, AJ, 122,2961

Salak, D., Nakai, N., Miyamoto, Y., Yamauchi, A., \& Tsuru, T. G. 2013, PASJ, 65, 66

Scannapieco, E., \& Brüggen, M. 2015, ApJ, 805, 158

Schaaf, R., Pietsch, W., Biermann, P. L., Kronberg, P. P., \& Schmutzler, T. 1989, ApJ, 336, 722

Schneider, E. E., \& Robertson, B. E. 2017, ApJ, 834, 144

Seaquist, E. R., \& Clark, J. 2001, ApJ, 552, 133

Smith, R., Flynn, C., Candlish, G. N., Fellhauer, M., \& Gibson, B. K. 2015, MNRAS, 448, 2934

Shopbell, P. L., \& Bland-Hawthorn, J. 1998, ApJ, 493, 129

Sofue, Y., Reuter, H.-P., Krause, M., Wielebinski, R., \& Nakai, N. 1992, ApJ, 395, 126

Strickland, D. K., \& Stevens, I. R. 2000, MNRAS, 314, 511

Strickland, D. K., \& Heckman, T. M. 2009, ApJ, 697, 2030

Telesco, C. M., Joy, M., Dietz, K., Decher, R., \& Campins, H. 1991, ApJ, 369, 135

Thompson, T. A., Fabian, A. C., Quataert, E., \& Murray, N. 2015, MNRAS, 449, 147

Thompson, T. A., \& Krumholz, M. R. 2016, MNRAS, 455, 334

Thompson, T. A., Quataert, E., Zhang, D., \& Weinberg, D. H. 2016, MNRAS, 455, 1830

van der Hulst, J. M. 1979, A\&A, 75, 97

Veilleux, S., Cecil, G., \& Bland-Hawthorn, J. 2005, ARA\&A, 43, 769

Veilleux, S., Rupke, D. S. N., \& Swaters, R. 2009, ApJL, 700, L149

Walter, F., Weiss, A., \& Scoville, N. 2002, ApJL, 580, L21

Walter, F., Brinks, E., de Blok, W. J. G., et al. 2008, AJ, 136 2563-2647

Wang, B. 1995, ApJL, 444, L17

Wang, B. 1995, ApJ, 444, 590

Werk, J. K., Prochaska, J. X., Cantalupo, S., et al. 2016, ApJ, 833,54

Westmoquette, M. S., Gallagher, J. S., Smith, L. J., et al. 2009, ApJ, 706, 1571

Westmoquette, M. S., Smith, L. J., \& Gallagher, J. S., III 2011, MNRAS, 414, 3719 
Young, J. S., \& Scoville, N. Z. 1984, ApJ, 287, 153

Yun, M. S., Ho, P. T. P., \& Lo, K. Y. 1993, ApJL, 411, L17

Yun, M. S., Ho, P. T. P., \& Lo, K. Y. 1994, Nature, 372, 530

Zhang, D., Davis, S. W., Jiang, Y.-F., \& Stone, J. M. 2017, arXiv:1708.02946 